\title{
Cytomegalovirus (CMV) infection and early onset pre-eclampsia.
}

\author{
Roya Sherkat $^{1}$, Behrouz Attaei ${ }^{2}$, Neda Pari Zangeneh ${ }^{3}$, Saba Sheikhbahae ${ }^{1 *}$, Somayeh Najafi ${ }^{1}$, Azar \\ Danesh $^{4}$, Majid Yaran', Maryam Nasirian ${ }^{2}$ \\ ${ }^{1}$ Acquired immunodeficiency research center, Isfahan University of Medical Sciences, Isfahan, Iran \\ ${ }^{2}$ Infectious Diseases and Tropical medicine research center, Isfahan University of Medical Sciences, Isfahan, Iran \\ ${ }^{3}$ Department of Infectious Diseases, Al-Zahra Hospital, Isfahan University of Medical Sciences, Isfahan, Iran \\ ${ }^{4}$ Obstetrics and Gynecology department, Isfahan University of Medical Sciences, Isfahan, Iran \\ ${ }^{5}$ Nosocomial Infection Research Center, Isfahan University of Medical Sciences, Isfahan, Iran
}

\begin{abstract}
Introduction: Pre-eclampsia is one of the common gestational disorders characterized by hypertension after 20 weeks of gestation and proteinuria with unknown different etiologies. It is a major cause of maternal and perinatal morbidity and mortality. Human Cytomegalovirus (CMV) can affect uterine villuses and maternal spiral arteries. Therefore this study was designed to evaluate the potential link between Cytomegalovirus (CMV) infection and early onset pre-eclampsia.

Materials and Methods: Thirty nine pregnant women with pre-eclampsia and forty healthy pregnant woman (as control group) were compared with each other in the term of demographic features and serum levels of IgM and IgG anti-CMV with an enzyme linked immunosorbent assay (ELISA). Also the avidity index for CMV IgG were evaluated and compared between two groups.

Results: All the members of case and control groups were seronegative for anti-CMV IgM. Anti-CMV IgM and anti-CMV IgG status did not significantly differ between two groups $(P=0.054$ and $P=0.151$, respectively). Both the mean value of anti-CMV IgM serum levels and anti-CMV IgG avidity were not statistically different in patients with pre-eclampsia in comparison with control group ( $\mathrm{P}=0.133$ and $P=0.238$ respectively). Although all the enrolled individuals had positive anti-CMV IgG status, CMV IgG serum titer was lower in patients with pre-eclampsia compared to healthy subjects $(174.35 \mathrm{vs}$. 208.65 Ru/ml, $\mathrm{P}=0.026$ ).

Conclusions: Lower level of anti-CMV IgG serum level in patients with pre-eclampsia represents decreased immune response to CMV infection which might have led to reactivation of chronic CMV infection and uterus vascular injury in the course of pregnancy.
\end{abstract}

Keywords: Cytomegalovirus (CMV), Pre-eclampsia, IgM and IgG anti-CMV, IgG anti-CMV avidity, Early onset preeclampsia (EOPE).

Accepted on August 02, 2018

\section{Introduction}

Pre-eclampsia is one of the most common gestational disorders which is seen in $2 \%-10 \%$ of pregnancies [1]. It is characterized by hypertension and proteinuria, and it is a major cause of maternal and neonatal mortality and morbidity worldwide $[2,3]$. Some life threatening complications of pre-eclampsia have been recorded such as eclampsia, liver rupture, stroke, renal failure and edema in mother, fetal growth restriction and pre-term birth in newborn [4]. The risk factors for preeclampsia are not completely understood and different theories have been proposed for it [5]. Some of these risk factors are diabetes, obesity, multiple pregnancies, primiparity, personal or family history of pre-eclampsia, and chronic hypertension [6-8].

Human Cytomegalovirus (CMV) is a DNA herpesvirus, which presents in infected nasopharyngeal secretions, urine, saliva, semen, cervical secretions, and blood. Up to $85 \%$ of women with low socioeconomic status and $50 \%$ of women with high socioeconomic status are seropositive by the time of pregnancy in developing countries [9]. CMV Infection impairs extravillous cytotrophoblasts migration and efficient invasion of uterine wall and maternal spiral arteries induced by chemokine CXCL12 [10]. There are antithesis results in the term of associations between CMV and pre-eclampsia. Study performed by Carmen et al. showed that CMV infection may be associated with pre-eclampsia but it is unlikely to be the triggering factor of pre-eclampsia [11]. While, Strand et al. [12] demonstrated that the presence of maternal antibodies to CMV was not associated with pre-eclampsia and CMV infection is unlikely to be a major cause of pre-eclampsia. Because of lack of adequate studies and the importance of surveillance to avoid adverse outcomes in pre-eclampsia and fetal or maternal death, 
Citation: Sherkat R, Attaei B, Zangeneh NP, et al. Cytomegalovirus (CMV) infection and early onset pre-eclampsia. Allied J Med Res. 2018;2(1):7-11.

this study was designed to evaluate the potential link between CMV infection and early onset pre-eclampsia.

\section{Materials and Methods}

This cross-sectional study was conducted in Obstetrics and Gynecology Department of Alzahra University Hospital, Isfahan, Iran from August 2015 to December 2015. Pregnant individuals with gestational age (GA) 20-34 weeks, who met the criteria of pre-eclampsia (EOPE)-blood pressure more than 140/90 $\mathrm{mmHg}$ and proteinuria-diagnosed by obstetrician were included in our study as case group. Patients with proven underlying disease such as any type of autoimmune disease, immunodeficiency disorders and/or infectious disease were excluded from the study. Patients with incomplete data were also count out. Pregnant healthy women with matched age and GA were enrolled as control group. A consent form has been signed to participate for all patients. The study received ethics approval from the Ethics Committee of Isfahan University of Medical Sciences (293386).
CMV serology of pregnant patients with early onset preeclampsia (case group) was compared with healthy pregnant individuals (control group). Blood samples for serum levels of IgM and IgG anti-CMV, IgG anti CMV avidity were obtained at the same peripheral vein puncture for all the individuals. $5 \mathrm{~mL}$ blood were obtained in gel tubes and allowed to clot for 30 minutes. Then blood samples centrifuged for 10 minutes at 2,500 rotations per minute, then freezed and stored at $-80^{\circ} \mathrm{C}$. IgM and IgG anti-CMV levels in the serum were measured with an enzyme linked immunosorbent assay (ELISA) using CMV IgM and IgG kit (Euroimmum from Germany). Results for CMV $\operatorname{IgG}$ and $\operatorname{IgM}$ were expressed as relative units per milliliter $(\mathrm{Ru} / \mathrm{mL})$.

The avidity index (AI) for CMV IgG was performed using Euroimmun kit and the formula $\mathrm{AI}=(\mathrm{OD}$ for the well washed with dissociating buffer/OD for the well washed with kit wash buffer) $\times 100$. CMV IgG avidity was expressed as a percentage.

Table 1. Studied variables in both control and pre-eclampsia groups.

\begin{tabular}{|c|c|c|c|c|c|}
\hline & \multirow{2}{*}{\multicolumn{2}{|c|}{ Variables }} & \multicolumn{3}{|c|}{ Group } \\
\hline & & & Pre-eclampsia & Control & $\mathrm{P}$-value \\
\hline \multicolumn{3}{|l|}{ Age (year) } & $29.79 \pm 6.62$ & $29.02 \pm 6.59$ & 0.606 \\
\hline \multicolumn{3}{|l|}{ GA (week) } & $29.71 \pm 4.83$ & $31.15 \pm 3.43$ & 0.135 \\
\hline \multirow{4}{*}{ Pregnancy history } & \multicolumn{2}{|l|}{ Gravid } & $2.2 \pm 1.1$ & $2.72 \pm 1.32$ & 0.062 \\
\hline & \multirow{2}{*}{ Abortion } & One & $8(21.6 \%)$ & $5(12.5 \%)$ & 0.645 \\
\hline & & Two & $2(5.4 \%)$ & $1(2.5 \%)$ & 0.424 \\
\hline & Death & $5(13.5 \%)$ & $5(12.8 \%)$ & 0.929 & \\
\hline \multicolumn{3}{|l|}{ Infertility history } & $9(23.1 \%)$ & $2(5 \%)$ & 0.02 \\
\hline \multicolumn{3}{|l|}{ Anti-CMV IgM (Ru/ml) } & $0.075 \pm 0.11$ & $0.041 \pm 0.07$ & 0.133 \\
\hline \multicolumn{3}{|l|}{ Anti-CMV IgG (Ru/ml) } & $174.35 \pm 55.18$ & $208.65 \pm 77.45$ & 0.026 \\
\hline \multicolumn{3}{|l|}{ Anti-CMV IgG avidity (\%) } & $73.92 \pm 13.2$ & $69.82 \pm 17.13$ & 0.238 \\
\hline \multirow{3}{*}{\multicolumn{2}{|c|}{ Anti-CMV IgM status }} & Negative & $30(76.9 \%)$ & $37(92.5 \%)$ & \multirow{3}{*}{0.054} \\
\hline & & Equivocal & $9(23.1 \%)$ & $3(7.5 \%)$ & \\
\hline & & Positive & 0 & 0 & \\
\hline \multirow{3}{*}{ Anti-CMV IgG status } & & High & $31(79.5 \%)$ & $26(65 \%)$ & \multirow{3}{*}{0.151} \\
\hline & & Intermediate & $8(20.5 \%)$ & $14(35 \%)$ & \\
\hline & & Low & 0 & 0 & \\
\hline
\end{tabular}

\section{Data Analysis}

Data were analyzed and reported only for patients who completed the trial. Statistical analysis of data was performed using SPSS version 22 software. To compare qualitative variables between groups Chi-square test was performed. The normal distribution of all studied parameters was checked with Kolmogorov-Smirnov test. Student t-test and paired t-test were used for variables which were distributed in a normal way, besides Mann-Whitney and Wilcoxon test were performed for variables that have not normal distribution. The two tailed $\mathrm{p}$ value $<0.05$ were considered significant.

\section{Results}

Forty-three patients were included in study. Among which 2 were dropped out due to autoimmune disease and 2 for infectious disease and finally, 39 patients completed the study. Demographic features in terms of age and GA in both groups were similar. Also, history of the number of gravid, abortion 
and death $(\mathrm{P}>0.05)$ were not different (Table 1). While, the rate of infertility history was higher in pre-eclampsia patients ( $23.1 \%$ vs. $5 \%, \mathrm{P}=0.02)$.

All members of case and control group have negative antiCMV IgM. Mean level of anti-CMV IgM was $0.075 \mathrm{Ru} / \mathrm{ml}$ in pre-eclampsia patients and $0.041 \mathrm{Ru} / \mathrm{ml}$ in control. Anti-CMV IgM serum levels did not significantly differ between two groups $(\mathrm{P}=0.133)$. Although all members of two groups have positive anti-CMV IgG status which means that all patients and healthy individuals were immune to the pathogen, CMV IgG serum titer was lower in patients with pre-eclampsia compared to healthy subjects (174.35 vs. $208.65 \mathrm{Ru} / \mathrm{ml}, \mathrm{P}=0.026)$.

Anti-CMV IgG avidity were not significantly different between two groups, however anti-CMV IgG avidity was higher in patients with pre-eclampsia. Anti-CMV IgG avidity was $73.92 \%$ in pre-eclampsia patients and $69.82 \%$ in control $(\mathrm{P}=0.238)$. Furthermore, anti-CMV IgM and anti-CMV IgG avidity status did not differ between two groups $(\mathrm{P}=0.054$ and 0.151 , respectively). As an accompanied finding infertility rate was significantly more in pre-eclampsia group than normal pregnancy control group $(23.1 \%$ in pre-eclampsia and $5 \%$ in normal pregnancy, $\mathrm{p}=0.02$ ).

\section{Discussion}

This study was performed to show the association of CMV infection and early onset pre-eclampsia. We examined three classes of CMV-specific serum antibodies IgG, IgM and IgG avidity, which were measured among patients with preeclampsia and matched healthy pregnant controls. Anti-CMV IgG indicates previous infection, anti-CMV IgM suggests acute or primary CMV infection and anti-CMV IgG avidity refers to how strongly an antibody binds to its antigen, and it is useful to distinguish CMV primary infection from non-primary infection and reactivation.

We found that although all patients and healthy individuals were immune to CMV, anti-CMV IgG serum levels were lower in pre-eclampsia patients compared to healthy subjects which was not similar to some previous published findings. Strand et al. demonstrated that the presence of maternal antibodies to CMV was not associated with pre-eclampsia [12]. In our study we evaluated the patients with early onset pre-eclampsia, while Strand et al. evaluated both patients with early and late onset pre-eclampsia. These differences between our and Strand results may due to different sample size and different methods. Xie showed that CMV infection seems to affect the occurrence of pre-eclampsia. They found that women with pre-eclampsia had increased anti-CMV IgG seropositivity with higher CMV IgG antibody level compared with normal pregnancy controls, while they did not find difference in anti-CMV IgM or IgA among studied groups [13]. Moreover, five years later, Xie et al. showed that early onset pre-eclampsia had significantly increased CMV IgG sero-positivity, up-regulated Toll-like receptor (TLR) -2/-4 mRNA expression, elevated serum IL-6 and TNF- $\alpha$, and reduced IL-10 compared with matched normal and non-pregnant controls, while they did not find significant difference between late onset pre-eclampsia and normal pregnancy controls [14]. Furthermore, Higgins et al. reported a case with CMV infection, who finally had early onset preeclampsia [15]. Dadelszen et al. showed that the anti-CMV antibodies was higher in early onset pre-eclampsia than in late onset pre-eclampsia, and normal pregnancy, which increase the risk for subsequent atherosclerosis as pathophysiology of early onset pre-eclampsia [16].

Several theses have been proposed to define the mechanisms of CMV infection in early onset pre-eclampsia, including endothelial-platelet dysfunction [17], acute atherosclerosis (leading uteroplacental ischemia) [18], inducing proinflammatory cytokines via up-regulated CD14, TLR-2 and TLR-4 (leading placental and fetal injury with liver and endothelial dysfunction) [19,20]. Moreover, up-regulated TLR expression plays an important role in apoptosis of trophoblast cells in the first trimester [21].

In contrast to these studies, and similar to our recent result, we previously found a significantly lower prevalence of serum anti-CMV antibodies in patients with recurrent spontaneous abortions (RSA) compared with pregnant female controls [22]. Also another survey found that the cytotoxic response to Staphylococcus enterotoxin B (SEB) antigen in patients with recurrent pregnancy loss (RPL) was significantly lower than control group [23]. In chronic or recurrent infection, detectable IgM is uncommon, while IgG levels may rise quickly [24]. On the other hand, in order to distinguish CMV primary infection from non-primary infection and reactivation, CMV avidity testing was suggested to perform [25]. So we checked antiCMV IgG avidity and found that all the healthy and preeclampsia patients are anti-cmv IgG positive and have high anti-CMV IgG avidity. Also we found that anti-CMV IgG level is lower in pre-eclampsia group than control group.

These results indicate that women with pre-eclampsia have some problems in immune responses to CMV infection, so decreased immune response to $\mathrm{CMV}$ infection may lead to reactivation of chronic $\mathrm{CMV}$ infection in the course of pregnancy, as it would induce CMV replication and host cell activation. Thus, CMV may influence vascular pathology by enhancing the inflammatory process and cellular activation responses [26]. Furthermore, pre-eclampsia may occur because of uterus vascular injury during pregnancy. But the role of viruses in induction of pre-eclampsia and whether women with pre-eclampsia have difficulty in responding to CMV, all needs more future researches. Also, we observed more infertility rate in EOPE cases.

\section{Conclusions}

Our results showed that Cytomegalovirus infection by itself is unlikely to be the risk factor of pre-eclampsia. Patients with pre-eclampsia had lower anti-CMV IgG serum levels compared to healthy pregnant women, maybe related to decreased immune response to $\mathrm{CMV}$ and reactivation of chronic infection which cause uterus vascular injury in the course of pregnancy. Also, we found that anti-CMV IgM serum levels and anti-CMV IgG avidity were not significantly different between two groups. More studies with greater number of pregnant women are required to verify if there is a relationship between Cytomegalovirus infection, reactivation 
Citation: Sherkat R, Attaei B, Zangeneh NP, et al. Cytomegalovirus (CMV) infection and early onset pre-eclampsia. Allied J Med Res. 2018;2(1):7-11.

of chronic CMV infection, cell-mediated immunity against CMV and early onset pre-eclampsia. Our study was unable to evaluate the effect of acute CMV infection on the rate of abortion as none of our patients had positive IgM. It is recommended to add a $3^{\text {rd }}$ group including positive anti-CMV IgM individuals for comparison.

\section{Conflicts of Interest}

The authors have indicated that they have no conflicts of interests regarding the content of this article.

\section{Acknowledgments}

This study was financially supported by Isfahan University of Medical, Isfahan, Iran Sciences. We gratefully acknowledge the dedicated efforts of the investigators, the coordinators, the volunteer patients who participated in this study.

\section{References}

1. Geographic variation in the incidence of hypertension in pregnancy. World Health Organization, International Collaborative Study of Hypertensive Disorders of Pregnancy. Am J Obstet Gynecol. 1988;158:80-3.

2. Mol BW, Roberts CT, Thangaratinam S, et al. Preeclampsia. Lancet. 2016;387:999-1011.

3. Say L, Chou D, Gemmill A, et al. Global causes of maternal death: a WHO systematic analysis. Lancet Glob Health. 2014;2:323-33.

4. Magee LA, Pels A, Helewa M, et al. Pregnancy Hypertens. 2014;4:105-45.

5. Conde-Agudelo A, Belizan JM. Maternal morbidity and mortality associated with interpregnancy interval: cross sectional study. BMJ. 2000;321:1255-9.

6. Mignini LE, Carroli G, Betrán AP, et al. Interpregnancy interval and maternal and perinatal outcome in 894,476 women: A multicountry sutdy. BJOG. 2015.

7. Shachar BZ, Lyell DJ. Interpregnancy interval and obstetrical complications. Obstet Gynecol Surv. 2012;67:584-96.

8. Barton JR, Sibai BM. Prediction and prevention of recurrent preeclampsia. Obstet Gynecol. 2008;112:359-72.

9. Cunningham GF, Leveno KJ, Bloom SL, et al. Chapter 64: Infectious diseases. Cytomegalovirus in Williams Obstetrics 24rd Edition, McGrawHill Med Ed. USA. 2014:Pp.1245-48.

10. Warner JA, Zwezdaryk KJ, Day B, et al. Human cytomegalovirus infection inhibits CXCL12-mediated migration and invasion of human extravillous cytotrophoblasts. Virol J. 2012;1:255.

11. Carmen R, Adina H, Rozalia G, et al. Cytomegalovirus Infection and Pre-Eclampsia. Acta Medica Marisiensis. 2016;62:280-4.

12. Strand KM, Odland ML, Iversen AC, et al. Cytomegalovirus antibody status at 17-18 weeks of gestation and pre-eclampsia: a case-control study of pregnant women in Norway. BJOG. 2012;119:1316-23.
13. Xie F, Hu Y, Magee LA, et al. An association between cytomegalovirus infection and pre-eclampsia: a casecontrol study and data synthesis. Acta Obstet Gynecol Scand. 2010;89:1162-7.

14. Xie F, von Dadelszen P, Nadeau J. CMV infection, TLR-2 and -4 expression, and cytokine profiles in early-onset preeclampsia with HELLPsyndrome. Am J Reprod Immunol. 2014;71:379-86.

15. Higgins L, Vause S, Tower C. Cytomegalovirus infection in association with early onset pre-eclampsia. BMJ Case Rep. 2010; bcr0320102803.

16. von Dadelszen P, Magee LA, Krajden M, et al. Levels of antibodies against cytomegalovirus and Chlamydophila pneumoniae are increased in early onsetpre-eclampsia. BJOG. 2003;110:725-30.

17. Reyna-Villasmil E, Mejia-Montilla J, Reyna-Villasmil N, et al. Endothelial microparticles in preeclampsia and eclampsia. Medicina clinica. 2011;136:522-6.

18. von Dadelszen P, Magee LA. Could an infectious trigger explain the differential maternal response to the shared placental pathology of preeclampsia and normotensive intrauterine growth restriction? Acta Obstet Gynecol Scand. 2002;81:642-8.

19. Lore K, Betts MR, Brenchley JM, et al. Toll-like receptor ligands modulate dendritic cells to augment cytomegalovirus- and HIV-1-specific T cell responses. J Immunol. 2003;171:4320-8.

20. Compton T, Kurt-Jones EA, Boehme KW, et al. Human cytomegalovirus activates inflammatory cytokine responses via CD14 and Toll-like receptor 2. J Virol. 2003;77:4588-96.

21. Abrahams VM, Mor G. Toll-like receptors and their role in the trophoblast. Placenta. 2005;26:540-7.

22. Sherkat R, Rostami S. Seropositivity of cytomegalovirus in women with unexplained recurrent spontaneous abortions. World Allergy Org J. 2007.

23. Tarokhian B, Sherkat R, Nasr-esfahani MH, et al. CD107a Expression and IFN- $\gamma$ Production as Markers for Evaluation of Cytotoxic CD3+ CD8+ T Cell Response to CMV Antigen in Women with Recurrent Spontaneous Abortion. 2014;7:323-30.

24. Friedman MG, aE AW. The use of serum $\operatorname{IgA}$ as a diagnostic marker in viral infections. Immunol Infect Dis. 1991;1:223-34.

25. Vauloup-Fellous C, Berth M, Heskia F, et al. Reevaluation of the $\operatorname{VIDAS}((\mathrm{R}))$ cytomegalovirus (CMV) IgG avidity assay: determination of new cut-off values based on the study of kinetics of CMV-IgG maturation. J Clin Virol. 2013;56:118-23.

26. Vossen RC, van Dam-Mieras MC, Bruggeman CA. Cytomegalovirus infection and vessel wall pathology. 1996;39:213-21.

\section{*Correspondence to:}

Saba Sheikhbahaei

Acquired Immunodeficiency Research Center, 
Isfahan University of Medical Sciences,

Isfahan, Iran
Email: sabash1368@gmail.com

Phone: +989356176162, Fax: +9832222811 